Marcos-Pablos, S., García-Holgado, A., \& García-Peñalvo, F. J. (2018). Trends in European research projects focused on technological ecosystems in the health sector. In F. J. García-Peñalvo (Ed.), Proceedings TEEM'18. Sixth International Conference on Technological Ecosystems for Enhancing Multiculturality (Salamanca, Spain, October 24th-26th, 2018) (pp. 495-503). New York, NY, USA: ACM. doi:10.1145/3284179.3284263

\title{
Trends in European research projects focused on technological ecosystems in the health sector
}

\author{
Samuel Marcos-Pablos \\ GRIAL Research Group, \\ Research Institute for Educational \\ Sciences, University of Salamanca \\ 37008 Salamanca, Spain \\ samuelmp@usal.es
}

\author{
Alicia García-Holgado \\ GRIAL Research Group, \\ Research Institute for Educational \\ Sciences, University of Salamanca \\ 37008 Salamanca, Spain \\ aliciagh@usal.es
}

\author{
Francisco José García- \\ Peñalvo \\ GRIAL Research Group, \\ Research Institute for Educational \\ Sciences, University of Salamanca \\ 37008 Salamanca, Spain \\ fgarcia@usal.es
}

\begin{abstract}
Over the past decade, the health domain has grown at a fast pace. The stakeholders are not only limited to patients, but also include formal and informal careers, doctors, research institutions and technologieal solution providers. As such, different technological ecosystems of interconnected health communities have arisen to adopt the best practices to improve the wellbeing and health of patients. In order to identify the lacks and opportunities in this area, this paper aims at providing a comprehensive overview of the ecosystems in the health domain, presenting a systematic mapping study of research projects developed in Europe and related to the field. The systematic mapping review was conducted on the AALProgramme, CORDIS and KEEP databases. The paper describes the methodology employed for conducting such a review, and provides an analysis of results that give an overview of the evolution of related European projects until today along with the conclusions obtained from the study.
\end{abstract}

\section{CCS CONCEPTS}

- Applied computing Health care information systems • Software and its engineering • Software and its engineering $\rightarrow$ Ultralarge-scale systems $\cdot$ Software and its engineering $\rightarrow$ Designing software

\section{KEYWORDS}

technological ecosystems, software ecosystems, European projects, health sector, systematic mapping, systematic literature review, European research.

\section{INTRODUCTION}

Technological ecosystems, also called software ecosystems (SECOs) in the literature $[1,2]$, have emerged in the last years as a solution to improve the knowledge management in heterogeneous contexts. They provide a general framework that allows defining and developing any type of technological solution in which data and information are the centre of the problem [3].

Technological ecosystems can be oriented to different domains, depending on the problems they solve. In particular, there is a research area related to use this technological approach in health sector.

On the other hand, the European Union has a strong investment in R\&D and demand side -measures in the health sector. Initiatives such as the European Innovation Partnership in Active and Healthy Ageing (EIP on AHA) to foster innovation and digital transformation in the field of active and healthy ageing [4], the Joint Programming on Neurodegenerative Diseases Research (JPND) or the Active and Assisted Living Programme (AAL), receive funding from the European Union, specifically, under grant agreements related to the Horizon 2020 call "Health, Demographic Change and Wellbeing".

In this context, the European Commission and other European entities have funded the definition and/or development of different technological ecosystems focused on improving different aspects in the health sector [5-7]. The analysis of these research projects will provide an overview of the current trends in the development of technological ecosystem in the health sector. Also, it can identify the lacks and opportunities to define new projects in this research area. 
The aim of this work is to conduct a systematic mapping study of research projects developed in Europe related to technological ecosystems in the health sector. The following sections describe the process undertaken, which follows the recommendations of Kitchenham [15] and Petersen [16] regarding the methodologies for conducting systematic literature reviews and mapping studies. The final goal is to identify gaps and research opportunities in the area, by formulating a set of relevant research questions and identifying and evaluating the related studies in the literature.

The paper has been organized in the following way. Section 2 describes the planning of the mapping process. Section 3 describes the extraction of the information. Section 4 presents the answers to the research questions. Section 5 analyses the results obtained during the mapping study. Finally, Section 6 summarizes the main conclusions of this work.

\section{MAPPING PLANNING}

In order to define the process to follow during the different stages, we identified the main aspects that were relevant in order to build the mapping protocol. This section lays out the results of said planning, including the research questions, the inclusion / exclusion criteria, the quality criteria and the followed search strategy.

\subsection{Identify the need of mapping}

First task before starting a systematic mapping or a systematic literature review is to identify the need of conducting the study. In this case, it is required to identify if there are other mapping studies about European research projects in the health sector focused on technological ecosystems.

Several searches were conducted in different types of sources. First, a search in Google and Google Scholar to find publications, reports or studies related to the European Union. On the one hand, a report about cross-border cooperation was found, in particular, a study to mapping EU-funded cross-border healthcare initiatives [8]. On the other hand, two papers were found. First, a paper that describes a systematic mapping study about European research on reducing social exclusion and stigma related to mental health [9]. Secondly, a paper about trends in ambient-assisted living platforms [10]. None of this works are focused on the same objectives that the study described in this paper.

Finally, we conducted a search in main scientific databases including: Web of Sciences (WoS), Scopus and PubMed. Only one paper found on Scopus could be related to this study but it compares the projects funded by the European Union about active aging and elderly's quality of life with projects funded by USA [11].

Thus, no systematic studies about European research projects in the health sector focused on technological ecosystems were found, so we think that conducting a systematic mapping is justified.

\subsection{Research questions}

The main objective of this study is to have an overview of the research trends related to technological ecosystems in the health sector focusing on European research projects. In particular, the systematic mapping aims to answer the following questions:

- MQ1: What are the trends in the development of technological ecosystems focused on health in Europe?

- MQ2: What is the application domain of the research conducted?

- MQ3: What types of institutions are inyolved in the project?

- MQ4: How are the stakeholders involved in the technological ecosystems developed?

- MQ5: Which calls fund this kind of research projects?

- MQ6: Which years cover the projects?

- MQ7: How much money was invested in these projects?

To define the scope of the review Petticrew and Roberts [12] propose the PICOC method:

- Population (P): European research projects focused on software ecosystems in the health sector.

- Intervention (I): conduct a systematic mapping about European research projects in the health sector that define and develop software ecosystems.

- Comparison (C): no comparison.

- Outputs (O): an overview about trends in technological ecosystems in health sector.

- Context (C): research contexts related to technological and software ecosystems in the health sector.

\subsection{Inclusion and exclusion criteria}

A set of inclusion and exclusion criteria was defined to select those works that are relevant to answer the defined research questions:

- IC1: The project is focused on software ecosystems or technological ecosystems in the health sector AND

- IC2: The project involves different European countries, namely, it is an international project AND

- IC3: The project is closed AND 
- IC4: The project is available in the most relevant databases supported by the European Union AND

- IC5: The project is classified in a call related to health or technology AND

- IC6: The information about the project is available in English.

Regarding the exclusion criteria, they are defined as follow:

- IC1: The project is not focused on software ecosystems or technological ecosystems in the health sector OR

- IC2: The project does not involve different European countries, namely, it is a national project OR

- IC3: The project is ongoing OR

- IC4: The project is not available in the most relevant databases supported by the European Union OR

- IC5: The project is not classified in a call related to health or technology OR

- IC6: The information about the project is not available in English.

\subsection{Quality criteria}

The inclusion and exclusion criteria allow to ensure a set of projects related to the scope of the mapping study, but they do not allow to ensure the quality of the results to answer the research questions proposed. To ensure this quality, a set of criteria was defined:

1. The website of the project is available?

2. The outputs of the project are available?

3. Are there more information in English available about the project than the project summary?

4. Are there scientific publications associated to the project?

5. Does the project provide a full definition of the ecosystem? (Implement part or the whole ecosystem)

6. Was the ecosystem developed? (Proposal, proof of concept or real system)

7. Is the project currently operating/available?

8. Does the ecosystem support evolution through the integration of new components?

\subsection{Search strategy}

There are a large number of entities that fund research projects in Europe, through different programmes and calls. The funded projects are published in different databases and webpages, but there is not a central database that collects all research projects, so it is necessary to identify the main databases.

First, the European Commission provides the Community Research and Development Information Service (CORDIS), the primary public repository and portal to disseminate information on all EU-funded research projects and their results (https://cordis.europa.eu).

Moreover, the European Commission co-financed European Innovation Partnerships (EIPs) in different areas, in particular, there is an EIP on Active and Healthy Ageing (https://ec.europa.eu/eip/ageing). This EIP identifies European Union funding sources which could be relevant on active and healthy ageing. One source was chosen to conduct this study, the Active and Assisted Living (AAL) programme which funds projects in public-private partnership in the field of information and communication technology (ICT) (http://www.aaleurope.eu).

Finally, the mapping study about cross-border cooperation in healthcare [8] identifies a set of online databases that cover a variety of projects related to healthcare: European Social Fund (ESF) (http://ec.europa.eu/esf/), European Regional Development Fund (ERDF) (http://bit.ly/2zrt9iD), CORDIS, KEEP Database (https://www.keep.eu/keep/search), CHAFEA Health Programmes database (http://bit.ly/2uijhlu) and EU Projects for Results Database (http://bit.ly/2L2Iurc).

A set of criteria was defined in order to choose the relevant databases for this study:

- It is a database focused on projects that involve several European countries.

- It is a reference database in the research scope.

- It allows using a search string equal or similar to the rest of the selected databases.

Finally, the sources chosen to conduct this study are: AAL Programme, CORDIS and KEEP. Both CORDIS and KEEP provide means to search their project database and filter the obtained results. On the other hand, the AAL programme does not provide with search tools and just contains a list of funded projects from the different programme calls. In this case, all funded projects were considered for further analysis.

Regarding the search terms employed in CORDIS and KEEP to identify solutions related to technological ecosystems or software ecosystems, only two terms have been chosen: platform and ecosystem. Several searches to select the right terms were conducted in the selected databases. For example, the combination of terms "software ecosystem", "technological ecosystem" or "digital ecosystem" provide scarce results in the health sector although they are implicitly part of the proposals, on the contrary, the platform term appears in many research projects to talk about ecosystems. Apart from the search strings, each database provides different search tools, with different filters. Moreover, there are databases that allow filtering by topics or categories.

Table 1 shows the terms and the filters used in each database. 


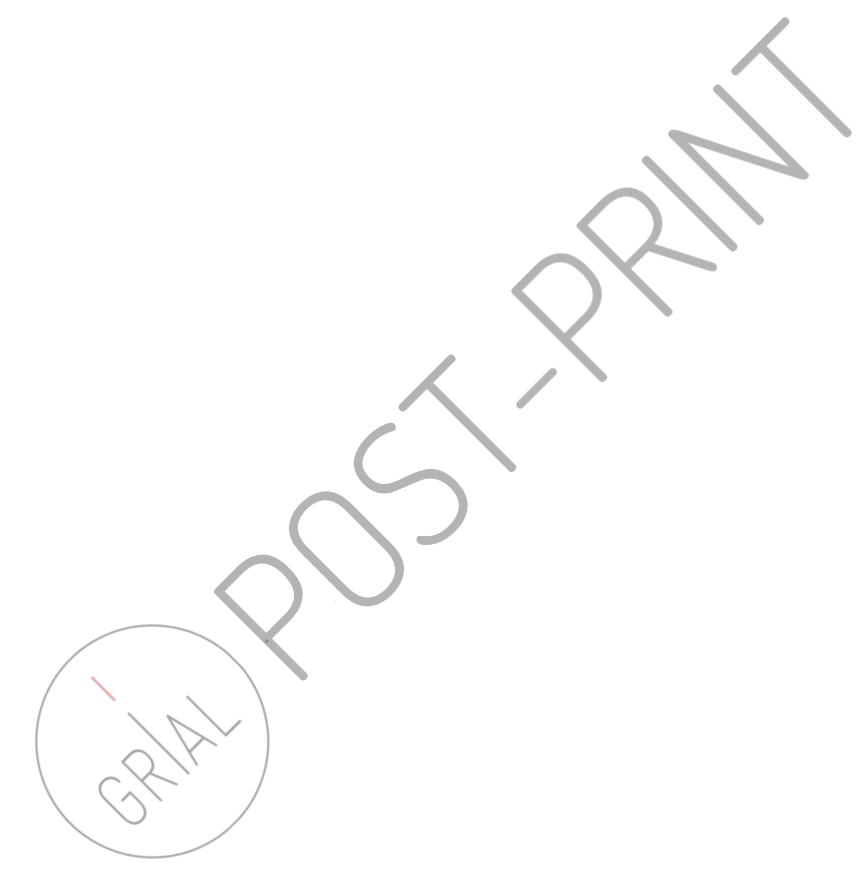


Marcos-Pablos, S., García-Holgado, A., \& García-Peñalvo, F. J. (2018). Trends in European research projects focused on technological ecosystems in the health sector. In F. J. García-Peñalvo (Ed.), Proceedings TEEM'18. Sixth International Conference on Technological Ecosystems for Enhancing Multiculturality (Salamanca, Spain, October 24th-26th, 2018) (pp. 495-503). New York, NY, USA: ACM. doi:10.1145/3284179.3284263

Table 1. Search strategies for each chosen database

\begin{tabular}{|c|c|c|c|c|}
\hline & AAL Programme & CORDIS & KEEP (I) & KEEP (II) \\
\hline Search terms & $\begin{array}{l}\text { All projects (there is no } \\
\text { search tool available) }\end{array}$ & platform or ecosystem & platform or ecosystem & $\begin{array}{l}\text { platform and health; } \\
\text { ecosystem and health }\end{array}$ \\
\hline Filters & $\begin{array}{l}\text { All projects (there is no } \\
\text { search tool available) }\end{array}$ & $\begin{array}{l}\text { Closed projects } \\
\text { Financing instrument: } \\
\text { Project } \\
\text { Subjects: Healthcare } \\
\text { delivery/services; Medicine } \\
\text { and Health }\end{array}$ & $\begin{array}{l}\text { Closed projects } \\
\text { Thematic: Health and } \\
\text { social services }\end{array}$ & $\begin{array}{l}\text { Closed projects } \\
\text { Thematic: ICT and } \\
\text { digital society }\end{array}$ \\
\hline
\end{tabular}

\section{DATA EXTRACTION}

After conducting the project search in the considered databases as described in the previous section, the selection of projects to be considered for the mapping study was performed in basis of the following steps:

1. The different project search results were collected in a spreadsheet (http://bit.ly/2ul21wz), removing all the duplicates across the databases.

2. The resultant projects were analyzed based on the inclusion/exclusion criteria (http://bit.ly/2KWMfSU). An initial title and project description screening was performed, in order to identify potentially eligible articles for inclusion in the following steps. However, as a result of employing heterogeneous databases, a slightly different approach was needed for each of them in order to access the individual project information. For example, in the AAL Programme and the KEEP database the project summary is only available in its associated webpage, so it was necessary to iteratively navigate through every project description page and manually download the corresponding project summary. In the case of the CORDIS database, search results can be conveniently downloaded in CSV (comma-separated values) format, but the resultant obtained file only contains a brief truncated description of each project. In this case, an initial screening was performed on the downloaded spreadsheet and, in those cases where the title and small description were not sufficient to make a decision, the authors quickly accessed the page with the entire content of the project description, and downloaded said complete description for the considered eligible projects for future analysis.

3. The selected projects then passed a quality assessment checklist applying the quality criteria defined during the planning stage and the information was collected in another spreadsheet (http://bit.ly/2uyLeWn). This categorization of the fulfillment of the quality criteria was performed according to the following scoring: Yes $=1$ point, Partially $=0.5$ points or No $=0$ points. In order to apply the quality criteria, we first considered the project final report in both the project page included in the databases or in the project own webpage (if available). In case we were not able to find the final report or it did not include enough information to answer the criteria, intermediate deliverables and reports were considered. If none of the above could be retrieved, a fast Google search based on the project title and / or acronym was performed in order to seek for additional sources of information such as scientific publications. In this case, only the first page of results was considered. Finally, those quality criteria which were not inferable from any of the above sources were marked as unanswered with the marker: "-"and given a 0 score.

After applying the above process the projects that obtained a score equal or higher of 6 out of 8 were collected in another spreadsheet (http://bit.ly/2NQM5dJ) for the final analysis. That threshold was selected in order to avoid low related projects or projects with just partial information available. Numerically:

- After applying the search strings in each source, 718 projects were collected, of which 203 are from AAL Programme, 81 from KEEP database and 434 from CORDIS database.

- $\quad$ After removing duplicates there are 707 projects.

- Once the inclusion and exclusion criteria were applied to title and abstract, there are 102 projects $(13,43 \%$ of the unique projects retrieved).

- $\quad$ Finally, a total of 19 projects were analysed (2,69\% of the unique projects retrieved). 


\section{RESULTS}

The extraction process described in the previous section provided the necessary data to answer the mapping questions. To analyse the data, a Jupyter notebook (http://jupyter.org) in Python was created as technical support of this process [13]. The notebook extracts the data from the spreadsheet in Google Sheets (http://bit.ly/2NQM5dI). This script is based on the work developed by Cruz-Benito http://bit.ly/2tS9JgF.

\subsection{MQ1: What are the trends in the development of technological ecosystems focused on health in Europe?}

Although the different project calls can mark the trend of the respectively funded projects, different types of technologies are employed to deploy the proposed ecosystems. Table 1 shows what are the main technologies involved in establishing relationships between the different ecosystem actors. It can be observed that web based ecosystems are the most frequently developed, and that a combination of sensors, mobile and cloud technologies are employed in the rest of the proposals.

Table 1. Type of ecosystem technology for the 19 considered projects

\begin{tabular}{|l|c|}
\hline Type of ecosystem technology & Count \\
\hline Web based & 10 \\
\hline Sensors + mobile + tv & 2 \\
\hline Web + sensors & 1 \\
\hline Sensors + mobile & 1 \\
\hline Web + mobile & 1 \\
\hline Sensors+robots+mobile+cloud & 1 \\
\hline Sensors + cloud & 1 \\
\hline Smart-TV & 1 \\
\hline Web+mobile+sensors & 1 \\
\hline
\end{tabular}

\subsection{MQ2: What is the application domain of the research conducted?}

Figure 1 answers the mapping question MQ2 in a graphical way. It can be seen that the main focus of the projects is in the ageing-well domain (6), followed by healthcare (5) and independent living (4). Other domains include biotechnology (2), assistive services (1), nutrition (1), rare diseases (1) an rehabilitation (1)

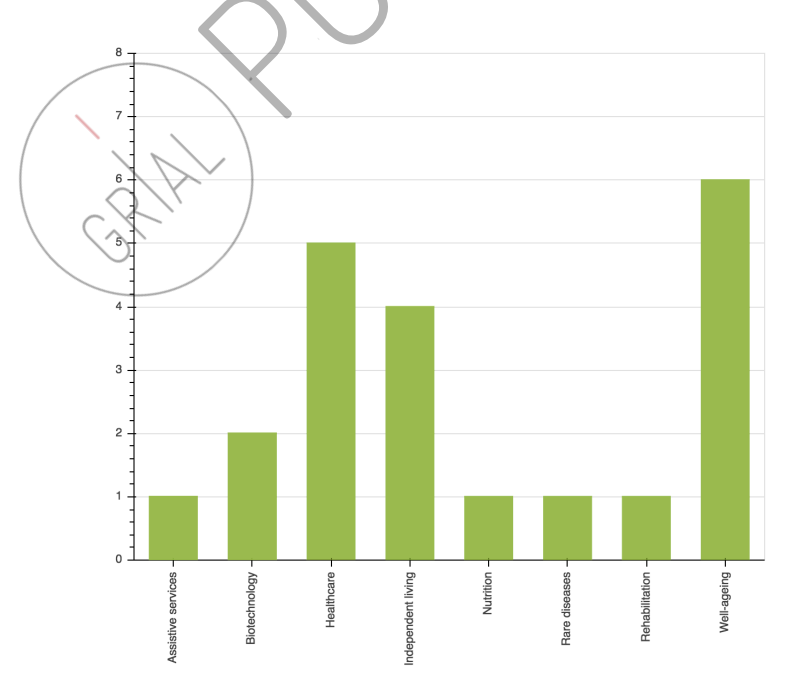

Figure 1. Number of projects per each domain. Note that some projects belong to more than one application domain.

\subsection{MQ3: What types of institutions are involved in the project?}

To answer the third mapping question, the institutions were classified in six categories: R\&D (universities, research centres, etc.), SME (small and medium enterprises), End-user (associations, care homes, NGOs, etc.), Business (big companies, hospitals, etc.), Multi- 
stakeholder. Figure 2 shows the distribution of institutions per category. There is total of 211 institutions involved in the selected projects.

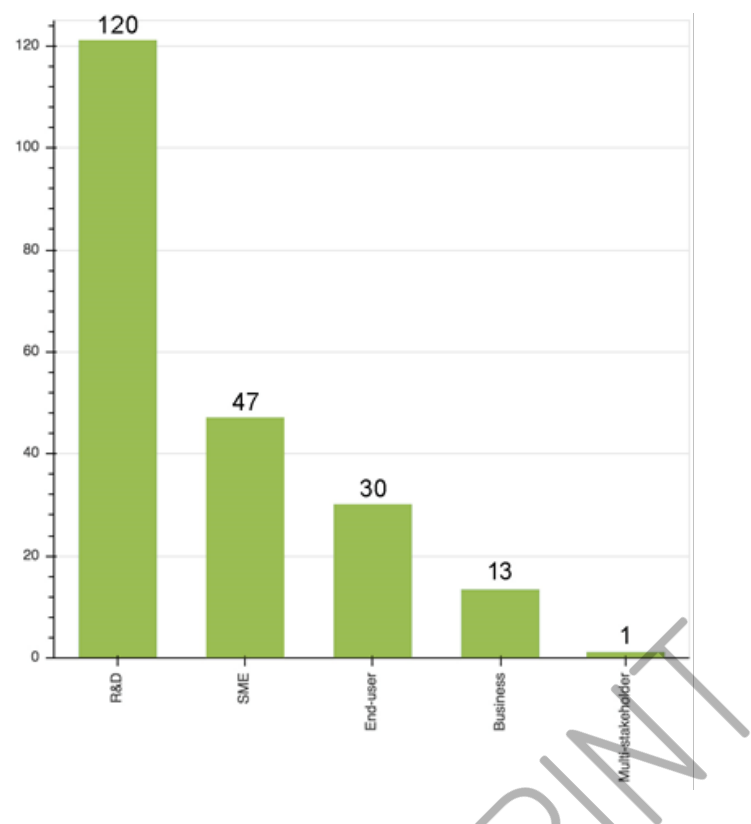

Figure 2. Total count of the different involved institutions along the 19 projects organized by type

\subsection{MQ4: How are the stakeholders involved in the developed technological ecosystems?}

Table 2 shows the obtained results regarding this mapping question. It can be observed that the majority of end-user related stakeholders correspond to institutions dedicated to provide different services to older persons (11) and informal carers (6). Related to the health sector, solutions engaging Pharmaceuticals (4), patients suffering different diseases (4) and Doctors (3) are the most common. As for the technology sector the main stakeholders consist in software providers/developers (7) and ICT solution providers (2)

Table 2. Total count of the different stakeholders involved in the ecosystems along all the projects

\begin{tabular}{|l|l|}
\hline Type & Count \\
\hline Older persons & 11 \\
\hline Informal carers & 6 \\
\hline Software provider & 7 \\
\hline Patients & 4 \\
\hline Pharmaceutical & 5 \\
\hline Doctors & 3 \\
\hline Scientists & 3 \\
\hline ICT solution provider & 2 \\
\hline Medical Equipment & 2 \\
\hline Formal carers & 2 \\
\hline Health device companies & 2 \\
\hline Business & 1 \\
\hline SMEs & 1 \\
\hline Consultancy & 1 \\
\hline Care planner & 1 \\
\hline Nutritionist & 1 \\
\hline Nurse & 1 \\
\hline Physician & \\
\hline Consulting & 1 \\
\hline Organizations & \\
\hline
\end{tabular}

\subsection{MQ5: Which calls fund this kind of research projects?}


Figure 4 shows a circle chart representing the percentage of projects found in each programme. It can be seen that the AAL programme covers the $73.68 \%$ of the total found projects in its different calls, whereas the $15.79 \%$ of the projects correspond to the FP7 ICT and HEALTH calls, and the $10.53 \%$ corresponds to the FP6-LIFESCIHEALTH programme.

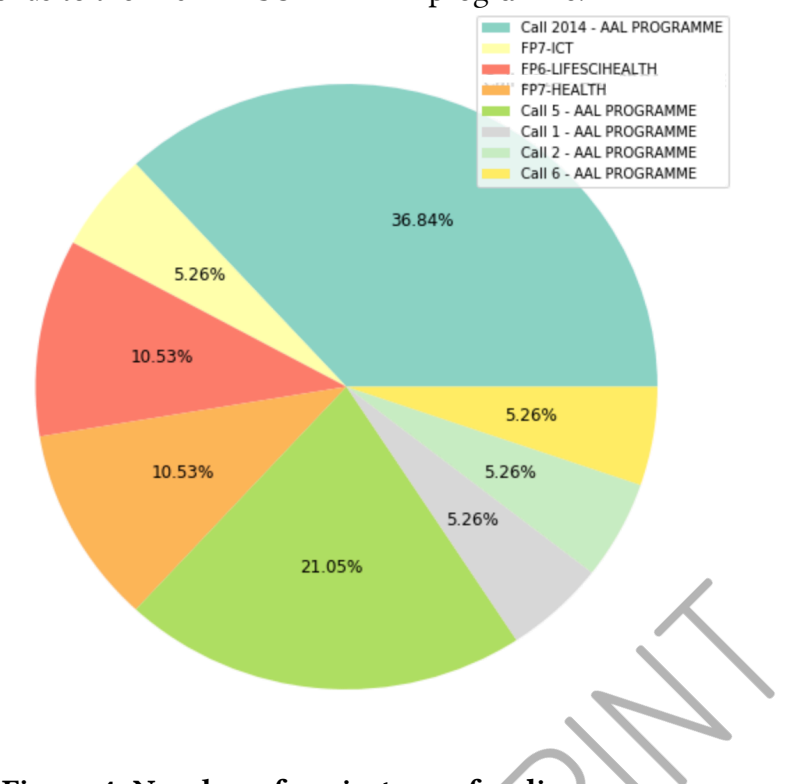

Figure 4. Number of projects per funding programme

\subsection{MQ6: Which period of time cover the projects?}

Figure 5 shows the distribution of the selected projects over time. It can be seen that the average project duration corresponds to 3-4 years, and that the majority of the projects are located in the 2010 to 2018 period.

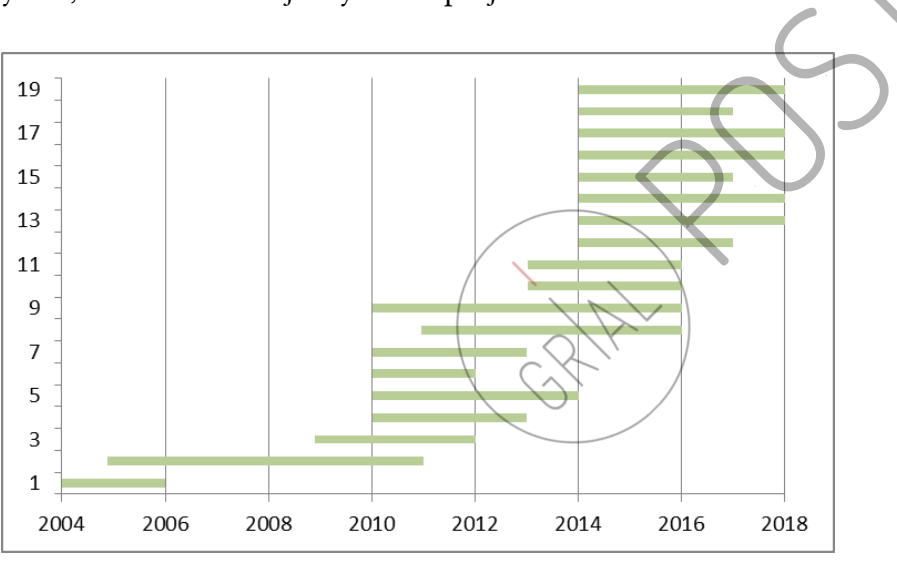

Figure 5. Distribution of the 19 projects over time. The ' $y$ ' axis represents the project number as coded in the spreadsheet

\subsection{MQ7: How much money was invested in these projects?}

To answer this mapping question Table 3 and Table 4 show the total investment per programme and per year along with the number of projects associated to the different calls and to the corresponding starting dates. Figure 6 graphically shows the overall yearly investment distribution. As a total, 67.470.946€ were provided for projects related to ecosystems in healthcare.

Table 3. Total investment per programme

\begin{tabular}{|l|c|c|}
\hline Programme & $\begin{array}{c}\text { Investment } \\
\text { Projects } \\
(\mathrm{N}=\mathbf{1 9})\end{array}$ \\
\hline FP6-LIFESCIHEALTH & $9.367 .500 €$ & 2 \\
\hline
\end{tabular}




\begin{tabular}{|l|l|l|}
\hline FP7-ICT & $14.631 .107 €$ & 3 \\
\hline FP7-HEALTH & $23.993 .478 €$ & 2 \\
\hline Call 1 - AAL Programme & $1.987 .368 €$ & 1 \\
\hline Call 2 - AAL Programme & $1.500 .000 €$ & 1 \\
\hline Call 5 - AAL Programme & $3.065 .214,60 €$ & 2 \\
\hline Call 6 - AAL Programme & $1.333 .205,66 €$ & 1 \\
\hline $\begin{array}{l}\text { Call 2014 - AAL } \\
\text { Programme }\end{array}$ & $11.593 .072,19 €$ & 7 \\
\hline
\end{tabular}

Table 4. Total investment per year

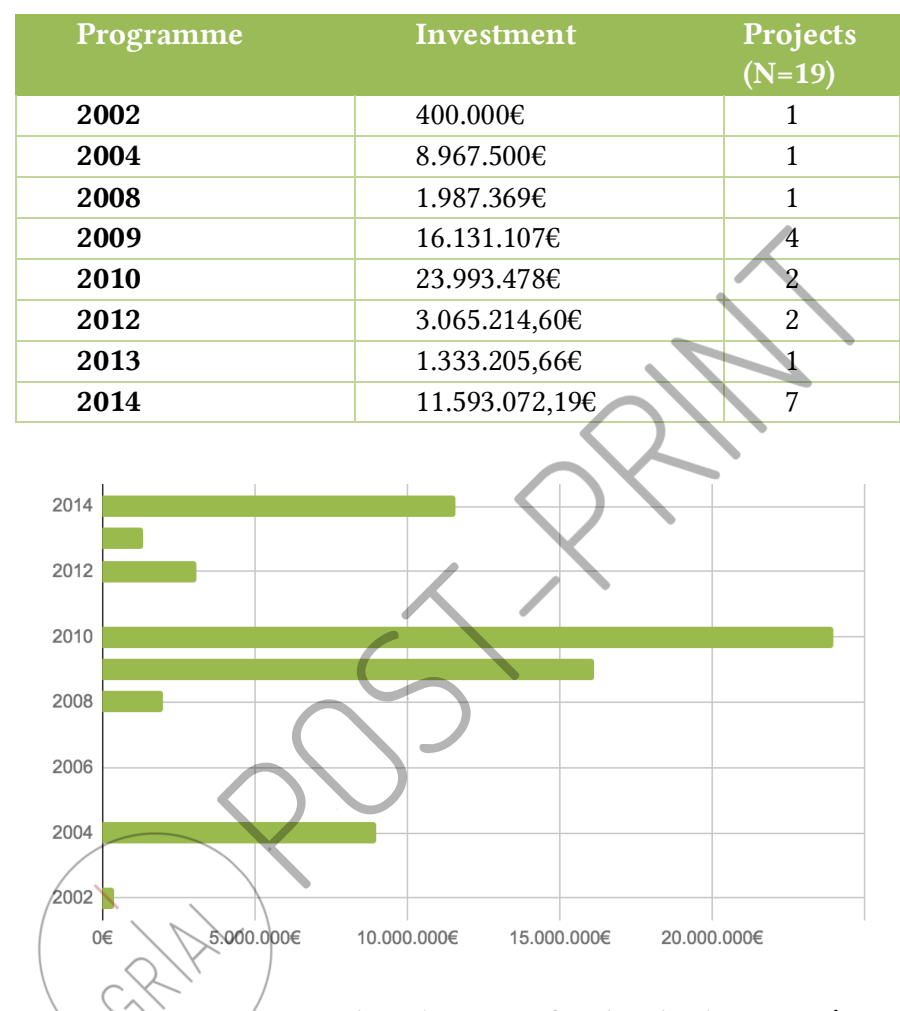

Figure 6. Total investment per year computed as the sum of individual projects' investment for each year

\section{DISCUSSION}

In this section, we will discuss each of the answers to our mapping questions. As many of the obtained results are interrelated, and the following discussion is better understood taking into account the nature of the programs funding the obtained projects, we will start the discussion by the fifth mapping question regarding the calls the obtained research projects belong to. It can be seen that the majority of obtained results (73.68\%) belong to the AAL programme in its different calls. The AAL programme's primary aim is to improve the living conditions of elderly people, while strengthening the international industrial opportunities for SMEs in the area of ICT. Next, 2 of the considered projects belong to the health section of the FP7 programme, which main focus was the translational research (i.e. the translation of basic discoveries in clinical applications) and strengthen the competitiveness and innovative capacity of European healthrelated industries and businesses. Another 2 projects belong to the FP6- LIFESCIHEALTH programme which was primarily focused on promoting community research through the coordination between regional, national, European and international health frameworks. Finally, one project from the FP7 ICT programme passed the quality assessment criteria. The activities considered for this call areas such as communication networks, network and service infrastructure stability and security, personalised ICT systems and digital content management. .From the description of the obtained calls, it can be observed that to a greater or lesser extent all of them promoted the collaboration between different actors with different motivations in the health sector which, when performed over a technological platform in turn resembles any of the definitions given to technological ecosystems [1-3]. 
In terms of the trends in the development of technological ecosystems, considering the technological platform employed for facilitating the interaction of the different actors the results highlight an amount of solutions that propose web platform based ecosystems. On the other hand, most of the rest of ecosystems make use of sensors in combination of web, mobile or cloud solutions. All the later cases mainly belong to the AAL program where, rather than using available technological frameworks, many of the proposed solutions are customized with different level of integration with health standards, resulting in different proposed architectures and protocols.

The main hypothesis for the above results relies is the scopes of the EU calls, which in turn lead to the different domains the proposed solutions are framed in. In the case of the AAL program solutions are mainly focused on ageing-well, independent living nutrition and assistive services, whereas the FP7 and FP6 health related ecosystem are focused on healthcare solutions for diabetes, cardiovascular disease therapeutics, cardiac patients monitoring, oncology and genetics. Due to being more related to health care, the later proposed solutions implement either standard-based architectures that comply with the different health standards (HL7, ISO/IEEE 11073, IHE IDCO Profile), or are focused in building networks between healthcare stakeholders and thus make use of existing web technologies for building communication means between the different actors.

Regarding the type of partners involved in the projects, it can be observed how research institutions (120), SMEs (47) and end-user organizations (30) are the main institutions that participate in this type of proposals. However, although large companies take part in the projects but in lesser quantity (13), the number is remarkably high taken into account that they are not eligible for funding. These large companies are mainly composed by pharmaceuticals and software providers. Analysing the scope and outputs of the ecosystems they belong to, the main value propositions for this kind of actors relies on the fact that participating in a European project offers a certain number of other opportunities apart from funding such as integrate potential new markets, gain access to innovation technologies developed within the ecosystem or reinforce the brand image.

The stakeholders considered in this work include not only those that correspond to the institutions that take part in the proposal, but also those who might benefit or be interested in the solutions developed in the projects. Regarding the nature of these stakeholders and end-users, they correspond to older persons, informal carers, pharmaceuticals, patients suffering different diseases, doctors and scientists. As for the technology sector the main stakeholders consist in software providers/developers and ICT solution providers. In terms of the particular scope of the proposed ecosystems, those related to the AAL program are clearly biased to senior users and formal and informal carers, whereas doctors, pharmaceuticals and patients are mostly found in the FP 6 and 7 projects.

Regarding the distribution of projects over time, ecosystem related projects have been developed between 2009 and 2018 . Prior to that, two projects that belonged to the FP6 programme implemented an ecosystem related to healthcare. In both cases they consisted in large networks of R\&D institutions over a web-based platform that provided services for enrolment of patients in clinical research, a suite of data analysis tools and protocols as well as a huge database of shared data between the participants. Even though the average duration of the projects is 3years, there is one particular FP6 project related to oncology - biological data that extended during 6 years and which results and services are still available. Call 1 and 2 of the AAL programme and the FP7 projects started in 2009-2010, whereas from 2013 only AAL health related ecosystem projects are found. The overall distribution of projects implementing ecosystem solutions is consisted with the evolution of technological / software ecosystems over time [14], as the first definition was introduced in 2003. Later, the number of publications related to software ecosystems until 2015 had increased linearly since 2003, with two peaks in 2011 and 2014.

Also, the above results are related with the amount of dedicated resources for this kind of projects. However, FP7 projects launched around 2010 involved a greater number of participants, and as such their budget is way higher than in other project proposals. In fact the 7 out of 19 projects that belong to the sixth and seventh Framework Programme comprise more than the $70 \%$ of the total associated budget, while the remaining $30 \%$ has been dedicated to the AAL program in its different calls.

\subsection{Threats to validity}

It has to be noted that, as with any research method, there could be threats to its validity and limitations in the current mapping. The first threat is that the inclusion of all the relevant projects in the field of ecosystems in healthcare is not guaranteed. This threat was mitigated by combining different databases and manual searches. However, as can be depicted from the results, the number of projects that have reached the final stage after applying the quality criteria is quite low. The main reason from this is the scarce information that can be gathered once the projects have finished, even after applying different search strategies and looking for information from different sources as described in section 3. It should be noted that this does not mean that the projects analyzed have not been successfully carried out, but that the information relative to their outputs could not be accessed.

Also there are two threats to internal validity in this systematic mapping. The first threat is that most of the projects do not pro- vide accurate descriptions or references for the ecosystem they implement, as in most cases it is not the main objective of the project. The second threat is related to the identification of values for classification criteria which again was not obvious in many cases. For that reason, some of the retrieved information had to be inferred from different sources, discussed and analyzed closely by all of the authors of the paper.

Finally, a threat to validity which is specific for this particular research is the bias in the results that could be introduced due to the nature of the funding programs that guide the scope and goals of the financed research. Again, this was mitigated by searching in different EU programs and calls and including a wide range of funding periods. 


\section{CONCLUSIONS}

This paper presents a systematic mapping review in the domain of European research projects focused on technological ecosystems in the health sector, which has been performed to try to investigate and understand how the European health community interprets and addresses the emerging ecosystem approaches. The review was focused on seven mapping questions and the main findings are:

- most of the rest of ecosystems make use of web platforms, in many cases in combination of sensors, mobile or cloud solutions

- the solutions are mainly focused on focused on ageing-well, independent living and health-related research communities

- when the solutions are AAL centred often consist of ad-hoc architecture. On the contrary, health related projects usually make use of standard-based solutions;

- $\quad$ partners include research institutions, SMEs, end-user organizations and large pharmaceutical and ICT provider companies.

- $\quad$ Projects and associated budget cover the 2003 - 2018 period, with in 2010 in 2014 correlated with the dates of the project calls.

Finally, trying to apply traditional mapping methodologies employed in research fields in the scope of European projects turned out to be a difficult task, as it is particularly difficult to find the necessary information related to finished projects. In this sense, some initiatives are being carried out such as the new AAL Market Observatory commissioned by the AAL Programme. This project will run until December 2018 and will provide a review of trends and perspectives in the field of AAL, annual reports on market and investment information, and a searchable database of relevant technologies (http://www.aal-europe.eu/market-observatory-for-aal).

\section{ACKNOWLEDGMENTS}

This research work has been carried out within the University of Salamanca PhD Programme on Education in the Knowledge Society scope (http://knowledgesociety.usal.es) and was supported by the Spanish Ministerio de Educación, Cultura y Deporte under a FPU fellowship (FPU014/04783).

This work has been partially funded by the Spanish Government Ministry of Economy and Competitiveness throughout the DEFINES project (Ref. TIN2016-80172-R) and the Ministry of Education of the Junta de Castilla y León (Spain) throughout the T-CUIDA project (Ref. SA061P17).

\section{REFERENCES}

[1] D. G. Messerschmitt and C. Szyperski. 2005. Software ecosystem: understanding an indispensable technology and industry. MIT Press Books 1.

[2] K. Manikas and K. M. Hansen. 2013. Software ecosystems - A systematic literature review. Journal of Systems and Software 86, 5 (05/05/2013), 1294-1306. DOI:http://dx.doi.org/10.1016/j.jss.2012.12.026.

[3] F. J. García-Peñalvo. 2018. Technological ecosystems for enhancing the interoperability and data flows. Fournal of Information Technology Research 11, 1, vi-x.

[4] European Innovation Partnership on Active and Healthy Ageing. 2018.

[5] M. Mikalsen, S. Hanke, T. Fuxreiter, S. Walderhaug, L. W.M. Wienhofen, and N. Trondheim. 2009. Interoperability services in the MPOWER Ambient Assisted Living platform. Studies in Health Technology and Informatics 150, 366-370.

[6] Sten Hanke, Christopher Mayer, Oliver Hoeftberger, Henriette Boos, Reiner Wichert, Mohammed-R. Tazari, Peter Wolf, and Francesco Furfari. 2011. universAAL An Open and Consolidated AAL Platform. In Ambient Assisted Living, R. Wichert and B. Eberhardt Eds. Springer, Berlin, Heidelberg, 127-140. DOI:http://dx.doi.org/10.1007/978-3-642-18167-2 10.

[7] L. M. Camarinha-Matos, J. Rosas, A.X. Oliveira, and F. Ferrada. 2013. Care services ecosystem for ambient assisted living. Enterprise Information Systems 9, 5-6, 607-

633. DOI:http://dx.doi.org/10.1080/17517575.2013.852693. Safety.

[9] S. Evans-Lacko, E. Courtin, A. Fiorillo, M. Knapp, M. Luciano, A. L. Park, M. Brunn, S. Byford, K. Chevreul, A. K. Forsman, L. Gulacsi, J. M. Haro, B. Kennelly, S. Knappe, T. Lai, A. Lasalvia, M. Miret, C. O'sullivan, C. Obradors-Tarragó, N. Rüsch, N. Sartorius, V. Švab, J. Van Weeghel, C. Van Audenhove, K. Wahlbeck, and A. Zlati. 2014. The state of the art in European research on reducing social exclusion and stigma related to mental health: A systematic mapping of the literature. European Psychiatry 29, 6 (2014/08/01), 381-389. DOI:http://dx.doi.org/10.1016/j.eurpsy.2014.02.007.

[10] Costa Angelo, Julián Vicente, and Novais Paulo. 2017. Advances and trends for the development of ambient-assisted living platforms. Expert Systems 34, 2, e12163. DOI:http://dx.doi.org/doi:10.1111/exsy.12163.

[11] I. Kirilov, M. Atzeni, A. Perra, D. Moro, and M. G. Carta. 2018. Active Aging and Elderly's Quality of Life: Comparing the Impact on Literature of Projects Funded by the European Union and USA. Clinical Practice \& Epidemiology in Mental Health 14, 1-5. DOI:http://dx.doi.org/10.2174/1745017901814010001.

[12] M. Petticrew and H. Roberts. 2005. Systematic Reviews in the Social Sciences: A Practical Guide. Blackwell Publishing, Malden, USA.

[13] A. García-Holgado, S. Marcos, and F. J. García-Peñalvo. 2018. Code repository that supports the research presented in the paper "Trends in European research projects focused on technological ecosystems in health sector".

[14] Oscar Franco-Bedoya, David Ameller, Dolors Costal, and Xavier Franch. 2017. Open source software ecosystems. Inf. Softw. Technol. 91, C (November 2017), 160185. DOI: h

[15] B. Kitchenham, S. Charters 2015. Guidelines for conducting systematic mapping studies in software engineering. Inf. Softw. Technol. 64, C (August 2015), 1-18. ttps://doi.org/10.1016/j.infsof.2017.07.007

[16] Kai Petersen, Sairam Vakkalanka, Ludwik Kuzniarz, Guidelines for conducting systematic mapping studies in software engineering: An update, Information and Software Technology, Volume 64, 2015, Pages 1-18, 